DOI: http://dx.doi.org/10.15359/rgac.1-56.4

Revista Geográfica de América Central. Nº 56 ISSN 1011-484X, enero-junio 2016

pp. 93-111

\title{
IMPACTOS GEOMORFOLÓGICOS DEL TERREMOTO DE LIMÓN (1991; MS=7.5) Y CONSIDERACIONES PARA LA PREVENCIÓN DE RIESGOS ASOCIADOS EN COSTA RICA
}

\author{
GEOMORPHOLOGICAL IMPACTS OF LIMON \\ EARTHQUAKE (1991, MS = 7.5) AND CONSIDERATIONS \\ FOR PREVENTING RELATED RISKS IN COSTA RICA
}

\author{
Adolfo Quesada Román ${ }^{1}$ \\ Universidad Nacional de Costa Rica
}

\begin{abstract}
RESUMEN
El Terremoto de Limón $(\mathrm{Ms}=7.5)$ se presentó el 22 de abril de 1991 y tuvo su epicentro en las coordenadas $9.685 \mathrm{~N}$ y $-83.073 \mathrm{~W}$. El objetivo de esta investigación fue estudiar la relación de los procesos detonados por el sismo en el modelado del relieve en el área más afectada: la Cuenca Limón Sur. La metodología consistió en una revisión bibliográfica de los estudios técnicos así como científicos realizados sobre el desastre y el análisis de sus implicaciones geomorfológicas. Los efectos cosísmicos fueron el levantamiento tectónico, la licuefacción, deslizamientos, inundaciones y un aumento de la carga de sedimentos en el sistema fluvial. Este suceso fue una importante enseñanza para Costa Rica en términos de gestión de una emergencia de gran magnitud, además cimentó la base de incipientes medidas de prevención y mitigación del riesgo de desastres.
\end{abstract}

Palabras clave: Levantamiento tectónico, licuefacción, deslizamientos, riesgos naturales, Geomorfología

1 Académico de la Escuela de Ciencias Geográficas de la Universidad Nacional de Costa Rica (UNA). Correo electrónico: adolfo.quesada@gmail.com

Fecha de recepción: 25 de setiembre de 2015

Fecha de aceptación: 05 de noviembre de 2015 
Adolfo Quesada Román. Impactos geomorfológicos del terremoto de Limón (1991; ms=7.5) y consideraciones para la prevención de riesgos asociados en Costa Rica

DOI: http://dx.doi.org/10.15359/rgac.1-56.4

\begin{abstract}
The Limon Earthquake (Ms = 7.5) occurred on April 22, 1991 with its epicenter located at coordinates $9,685 \mathrm{~N}$ and $-83,073 \mathrm{~W}$. The objective of this research was to study the relationship of the processes triggered by the earthquake on modeling the topography of the most affected area -the Southern Limon Basin. The methodology consisted on a literature review regarding the technical and scientific studies conducted about the disaster and the analysis of its geomorphological implications. The coseismic effects that occurred were the tectonic uplift, liquefaction, landslides, floods, and an increase of the fluvial system's sediment load. This event was a great lesson for Costa Rica in terms of managing a major emergency, and it also helped building the foundation for emerging prevention measures and disaster risk reduction.
\end{abstract}

Keywords: Tectonic uplift, liquefaction, landslides, natural risks, Geomorphology

\title{
Introducción
}

Antes de 1991, para los especialistas de las Ciencias de la Tierra y el Código Sísmico de Costa Rica, la región Caribe tenía un potencial sísmico bajo. El análisis histórico e instrumental había mostrado importantes eventos sísmicos durante los siglos XIX y XX en este territorio, particularmente el terremoto de San Estanislao ocurrido el 7 de mayo de 1822, presentando un fallamiento inverso con epicentro y características similares al evento de 1991 (Boschini y Montero, 1994).

El 22 de abril de 1991 a las 21:56 horas (UTC) ocurrió el Terremoto de Limón, el cual tuvo su epicentro en el área de Limón - Pandora en las coordenadas geográficas: $9.685 \mathrm{~N} \mathrm{y}-83.073 \mathrm{~W}$ (figura 1). Este evento tuvo una magnitud de $7.5 \mathrm{M}_{\mathrm{s}}$ del cual resultaron 109 heridos, 7439 damnificados y 41 muertos. La máxima intensidad determinada fue de $\mathrm{X}$ en la escala de Mercalli modificada y las pérdidas económicas estimadas fueron de unos 43 millones de dólares (Obaldía et al., 1991; USGS, 2015).

El Terremoto de Limón causó afectación en un área aproximada de 8 $000 \mathrm{~km}^{2}$ que incluyeron el $80 \%$ del territorio de Costa Rica y un 20\% de Panamá, los mayores daños fueron en líneas vitales: carreteras, ferrocarriles, puentes, puertos y acueductos. Las pérdidas sumaron 21 991,9 millones de colones, equivalente a un 4,21\% del Producto Interno Bruto del país en ese momento. Durante la fase de atención de la emergencia, se tuvo como prioridad la asistencia humanitaria, la cual se enfatizó en la rehabilitación de infraestructura para lo cual se estableció un puente aéreo, una red de comunicaciones así como varias brigadas como salud, albergues y abastecimiento (Morales, 1994).

El área de ruptura del terremoto se localizó en la Cuenca de Limón (Figura 1), esta agrupación geológica consiste en una secuencia de rocas 
sedimentarias y volcánicas del Cenozoico, las cuales presentan una deformación representada en pliegues con ejes E-W y NW-SE y con fallas con rumbos N-S, E-W y NW-SE.

La ruptura primaria tuvo cuatro subeventos en un periodo de 25 segundos y un área de ruptura de 3200 a $4500 \mathrm{~km}^{2}$; donde el tamaño de la zona de ruptura, la complejidad del evento principal y la cantidad de réplicas indican que estructuras de fallamiento secundario pudieron estar asociados al plano de ruptura principal (Montero et al., 1991; Güendel et al., 1991; Rojas, 1991; Goes y Schwartz, 1991; Denyer et al., 1994a). La interpretación del mecanismo focal, el levantamiento de la línea de costa y el desplazamiento horizontal hacia el NE en los alrededores de Limón hacen suponer que el plano de falla principal tiene su traza bajo el mar (Denyer et al., 1994a).

Figura 1. Localización del epicentro del Terremoto de Limón del 22 de abril de 1991.

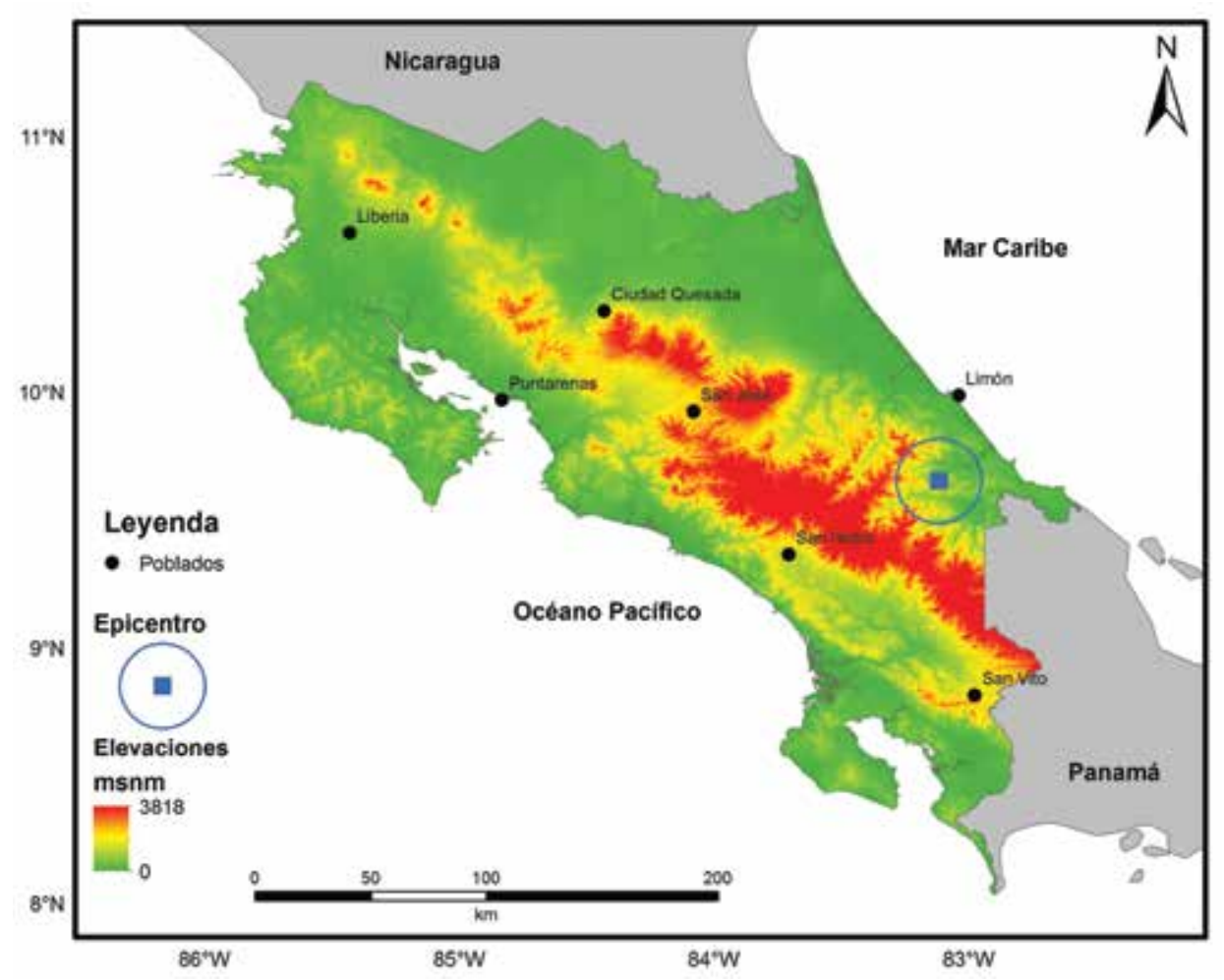

Fuente: ITCR (2014) y Denyer et al., 1994b. 
Adolfo Quesada Román. Impactos geomorfológicos del terremoto de Limón (1991; ms=7.5) y consideraciones para la prevención de riesgos asociados en Costa Rica

DOI: http://dx.doi.org/10.15359/rgac.1-56.4

\section{Características físico-geográficas del área de estudio}

La Cuenca Limón fue la zona que tuvo mayores efectos directos e indirectos a partir del Terremoto de Limón, es una depresión elongada que se extiende desde la plataforma marina hacia el continente en el SE de Costa Rica con unos cincuenta kilómetros de ancho, situada en la unidad morfotectónica del trasarco volcánico, el cual es producto del proceso de subducción entre las placas de Cocos y Caribe.

Su límite norte con la Cuenca Limón Norte, está definido por una serie de estructuras positivas enterradas, alineadas en dirección E-W y de edad Eoceno Medio Tardío, donde el levantamiento regional de la región costera Sur del Caribe revela que los estilos estructurales-tectónicos son diferentes entre las cuencas de Limón Norte y Limón Sur, cuyo límite se encuentra activo y es una extensión del Cinturón Deformado de Panamá que atraviesa el país en el Sistema de Falla Transcurrente de Costa Rica (Fernández et al., 1994; Mora y Valverde, 2005).

Además, el aporte sedimentario producto de la erosión desde la Cordillera de Talamanca y la Cordillera Volcánica Central, el desarrollo de ambientes someros de deposición y turbidez generaron a lo largo de 65 millones de años una serie de litologías que en la actualidad componen la Cuenca Limón. Entre ellas dominan las formaciones Tuis, Fila de Cal, Pelada, Barbilla, Senosri, Uscari, Río Banano, Quebrada Chocolate, Moín y Suretka (figura 2). 
Figura 2. Columnas estratigráfico-sedimentológicas modificadas de la cuenca Limón:
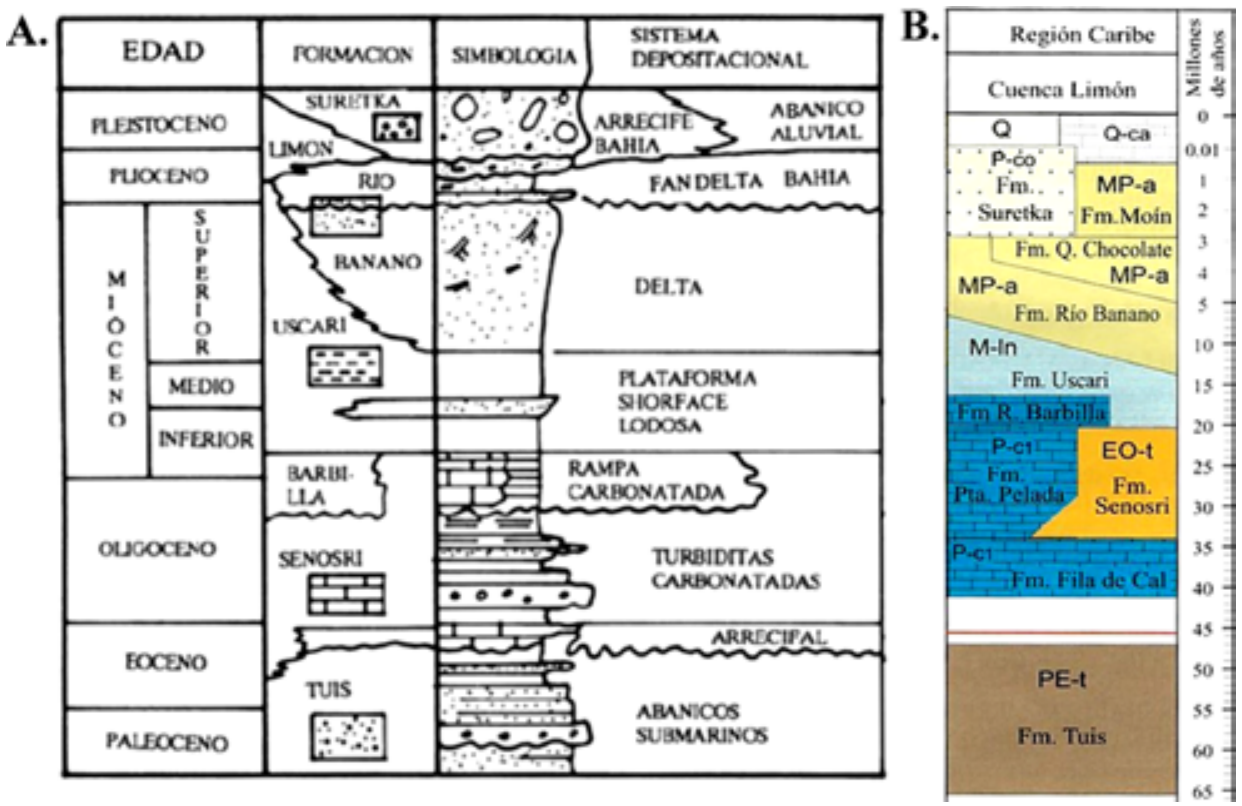

Fuente: A. Fernández et al., 1994 y B. Denyer y Alvarado, 2007.

En general, la conformación geológica de la Cuenca Limón se explica a través de los procesos tectónicos de deformación regional y local; y la sedimentación de las llanuras aluviales del Caribe costarricense debido a la erosión de los sistemas montañosos: Cordillera Volcánica Central y Cordillera de Talamanca, así como la integración de sustratos de ambientes de deposición somera a lo largo de la costa Caribe.

La dinámica interna y externa no pueden ser explicadas de manera separada, ya que una condiciona la evolución de la otra y por ende modela el relieve. Para ello, es fundamental explicar las características propias de esta región de Costa Rica a través de un análisis integral físico-geográfico, para lo cual se hace uso del concepto de provincias fisiográficas, las cuales son grandes unidades territoriales, que agrupan territorios homogéneos en cuanto a génesis, estructura, litología, morfología e incluso procesos de modelado en el relieve (Lugo, 2011).

En la regionalización fisiográfica también se consideran las condiciones climáticas, hidrografía, suelos y vegetación, desde el SE de México 
Adolfo Quesada Román. Impactos geomorfológicos del terremoto de Limón (1991; ms=7.5) y consideraciones para la prevención de riesgos asociados en Costa Rica

DOI: http://dx.doi.org/10.15359/rgac.1-56.4

hasta Panamá se suman quince Provincias Fisiográficas y en este marco Costa Rica está dividida en las siguientes: 8. Depresión de Nicaragua; 11. Frente Volcánico Chorotega; 12. Antearco Chorotega y 13. Trasarco Chorotega (Marshall, 2007) (figura 3).

Figura 3. Provincias fisiográficas de América Central y el SE de México

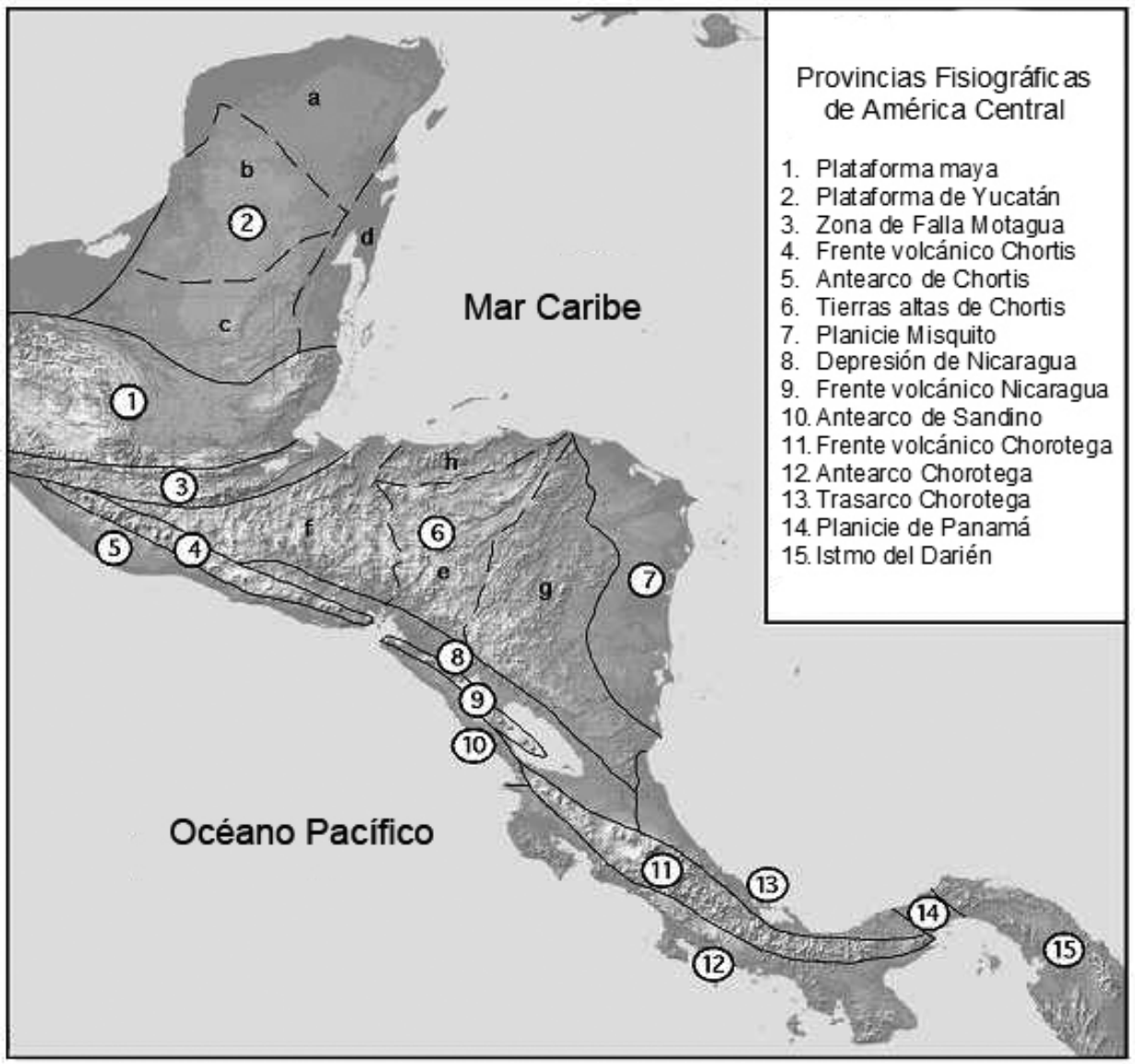

Fuente: Marshall, 2007 Modificado.

La Cuenca Limón está relacionada con el Trasarco Chorotega (figura 3: Provincia 13), el cual representa la vertiente Caribe de Costa Rica y por el SE tiene continuidad hacia Panamá. El relieve es de llanuras aluviales extensas que están vinculadas con los ríos Chirripó, Tortuguero, Reventazón, 
Pacuare, Matina, Madre de Dios, Moín, Banano, Bananito, La Estrella y Sixaola, todos nacen ya sea en la Cordillera Volcánica Central o de Talamanca; y desembocan en el Mar Caribe (Bergoeing, 2007; Bergoeing, 2013).

El clima que predomina es lluvioso durante todo el año, debido a la influencia de lluvias estacionales (mayo a noviembre) y frentes fríos (diciembre a febrero); por lo que las inundaciones son frecuentes en cualquier momento, pero se agudizan en temporada de lluvias extraordinarias. Los promedios anuales de precipitación están por encima de $3000 \mathrm{~mm}$ y las temperaturas medias, superan $\operatorname{los} 26^{\circ} \mathrm{C}$ (Solano y Villalobos, 2001).

Los suelos con mayor distribución son los Inceptisoles y Ultisoles, ambos presentan un grado avanzado de lixiviación de sus bases y desarrollan amplias cortezas de intemperismo, proceso que se ve favorecido por la baja inclinación del terreno (llanuras aluviales), condiciones climáticas estables (altas temperaturas y elevado régimen pluviométrico) y la acción del tiempo (Berstch, 1995; Buol et al., 2008).

En cuanto a la vegetación, existen los siguientes tipos de bosques: húmedos tropicales (Terminalia amazonia, Carapa guinensis); muy húmedos tropicales (Ceiba pentandra y Dipteryx panamensis) y muy húmedos premontano, donde prevalecen los ecosistemas perennifolios con árboles que pueden superar los $40 \mathrm{~m}$ de altura (Bolaños et al., 2005; Quesada, 2007).

Todas estas características físico-geográficas hacen de este territorio una zona única tanto en su geodinámica interna y externa, y tienen relación directa con la respuesta de este sistema natural ante umbrales geomorfológicos severos como puede serlo una alteración atmosférica drástica con el desarrollo de inundaciones y marejadas, o como lo fue el Terremoto de Limón de 1991.

\section{Metodología}

El marco metodológico de la presente investigación consta de tres fases: revisión bibliográfica, organización de la información, e impactos geomorfológicos (como resultados y discusión de este trabajo). Cada etapa tiene como labor imprescindible la de mejorar el entendimiento de la dinámica tectónica, geológica y geomorfológica del Caribe costarricense en función de las modificaciones acaecidas posterior al evento sísmico de 1991.

La primera fase consistió en la revisión bibliográfica, para ello se hizo una búsqueda exhaustiva de artículos científicos sobre el evento tanto 
Adolfo Quesada Román. Impactos geomorfológicos del terremoto de Limón (1991; ms=7.5) y

consideraciones para la prevención de riesgos asociados en Costa Rica

DOI: http://dx.doi.org/10.15359/rgac.1-56.4

a nivel nacional como internacional, se encontró un acervo importante en un número especial de la Revista Geológica de América Central de la Universidad de Costa Rica sobre el Terremoto de Limón publicado en 1994 donde se trataron desde temas geológicos, tectónicos, geomorfológicos, climáticos, biológicos, de infraestructura y sociales.

Asimismo, se ubicaron varios informes de la Comisión Nacional de Prevención de Riesgos y Atención de Emergencias (CNE), del Observatorio Sismológico y Vulcanológico de la Universidad Nacional de Costa Rica (OVSICORI) y otros estudios independientes enfocados en la vulnerabilidad social de la población afectada por el evento.

La segunda fase, de organización de la información, permitió generar una serie de fichas bibliográficas donde se condensaron los datos más relevantes de cada libro, artículo científico o informe que fuera importante retomar en el análisis; este proceso facilitó el entendimiento de las condiciones geofísicas (internas y externas) y sociales que conformaron un escenario de riesgo que dio paso a un desastre con las características del Terremoto de Limón.

La tercera fase metodológica corresponde a los impactos geomorfológicos, donde se estudiaron las respuestas morfológicas post-evento, desde el levantamiento de la línea de costa, la licuefacción en las llanuras aluviales de las cuencas hidrográficas próximas al epicentro y el disparo de cientos de procesos de ladera en las estribaciones montañosas al NE de la Cordillera de Talamanca, además de otros procesos asociados a posteriori del evento.

\section{Impactos geomorfológicos}

El terremoto de Limón de 1991 es un interesante ejemplo para entender las implicaciones de un sismo en la dinámica y evolución del relieve en un país tropical. Los procesos cosísmicos más representativos producto del evento fueron el levantamiento de la línea de costa, la licuefacción, los procesos de ladera (deslizamientos), las inundaciones posteriores, el aporte de sedimentos y modificación de la carga de los ríos (figura 4). 
Figura 4. Mapa integrado con los principales procesos cosísmicos del Terremoto de Limón de 1991: levantamiento

tectónico y procesos de ladera

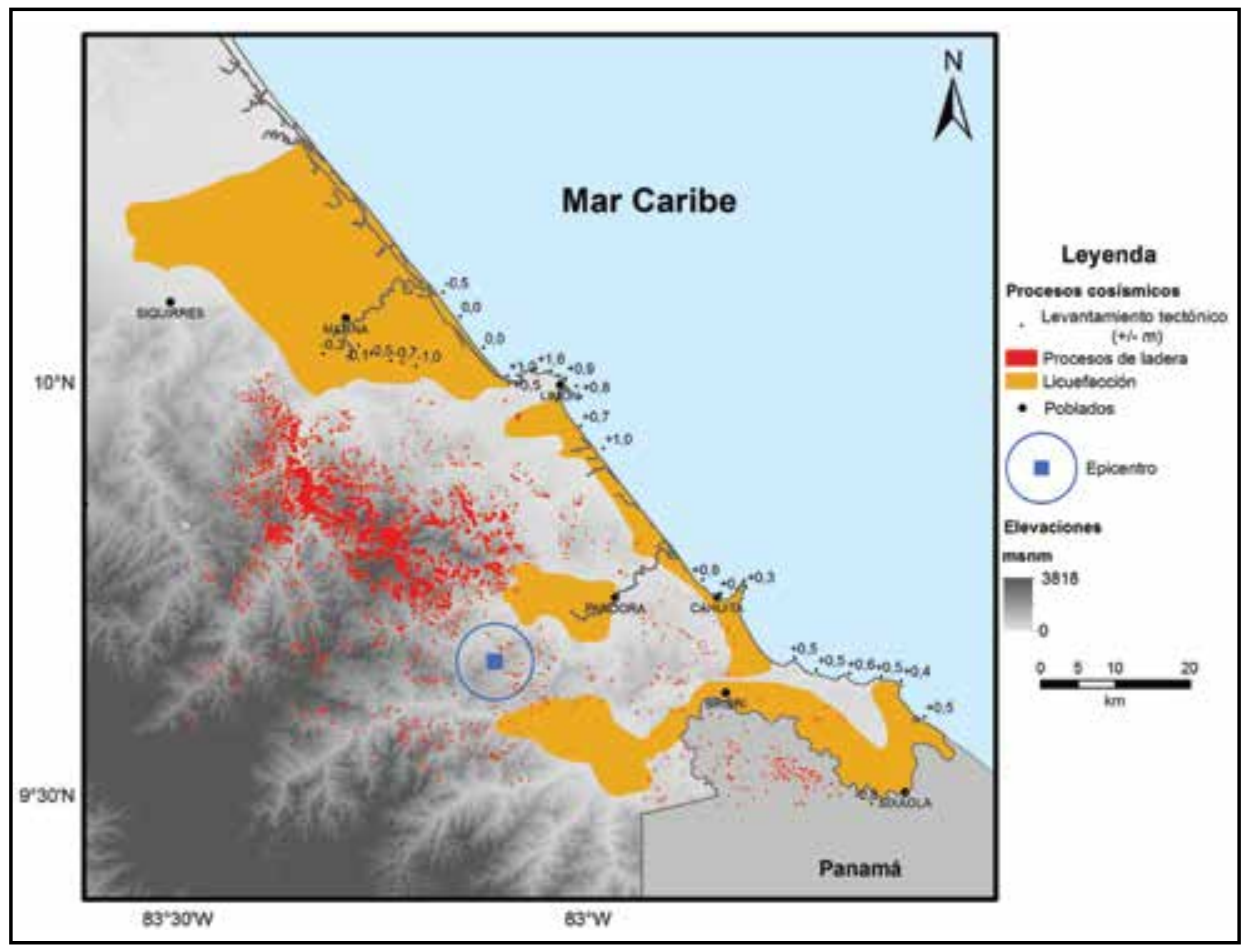

Fuente: Procesos de ladera (Modificado de Hernández et al., 1992), y licuefacción (Modificado de Denyer et al., 1994b).

El efecto tectónico más evidente y espectacular fue el levantamiento cosísmico de unos $100 \mathrm{~km}$ de la línea de la costa Caribe. La magnitud de dicho ascenso de la plataforma calcárea osciló entre 0.3 y $1.85 \mathrm{~m}$, el cual ocurrió en bloques separados por fallas transcurrentes de rumbo NE, hubo también levantamiento tierra adentro, con magnitudes superiores a los 3 metros (Denyer et al., 1994a, Denyer et al., 1994b).

Este proceso no fue continuo y varió de $40-50 \mathrm{~cm}$ cerca de río La Estrella, $40 \mathrm{~cm}$ en el Muelle Alemán y $2 \mathrm{~m}$ en Playa Bonita; las terrazas de la nueva línea de costa tuvieron valores diversos, de 15 a $20 \mathrm{~m}$ frente al Aeropuerto de Limón, de $30 \mathrm{~m}$ a la izquierda del río Banano y cerca de 50 $m$ en la boca del río La Estrella. El nuevo nivel de marea en playas rocosas 
Adolfo Quesada Román. Impactos geomorfológicos del terremoto de Limón (1991; ms=7.5) y

consideraciones para la prevención de riesgos asociados en Costa Rica

DOI: http://dx.doi.org/10.15359/rgac.1-56.4

presentó distancias muy variables alcanzando más de $60 \mathrm{~m}$ en Playa Bonita (Amador et al., 1994).

El estudio de Obaldía et al. (1991) indicó que los cambios de la línea de costa se produjeron en mayor magnitud en Limón y disminuyen hacia el noroeste y sureste de esta ciudad. Se encontró un máximo de $1.51 \mathrm{~m}$ de levantamiento entre el muelle de Moín y la playa Portete, y un mínimo de 0.36 m ubicado al oeste del cementerio de Limón.

Los cambios de pendiente como resultado del levantamiento cosísmico de esta área no son uniformes; no obstante, incidieron directamente en el régimen de drenajes y afectaron obras de infraestructura en que las modificaciones de nivel son importantes, hacia el noroeste de Limón, en el tramo Moín-Doce Millas el cambio del levantamiento decrece uniformemente; en contraste, hacia el sureste de Limón se encontró que la deformación cosísmica es positiva y disminuye en magnitud a medida que la nivelación se aleja de Limón.

De acuerdo con el análisis histórico, se infiere que se produjeron dos eventos anteriores a 1991, cuyas magnitudes fueron mayores a 7.0, los cuales se han presentado a lo largo del Cuaternario. Los levantamientos antes de 1991 parecen haber sido básicamente verticales sin deformación ni basculamientos como lo indican las terrazas horizontales a unos $50 \mathrm{~m}$ de elevación en la ciudad de Limón (Denyer et al., 1994a).

Como efecto paralelo al levantamiento de la línea de costa estuvo la afectación de organismos de la zona de entremareas; el sublitoral somero y los arrecifes coralinos, dichos ecosistemas quedaron expuestos y murieron poco después. En el Parque Nacional Cahuita se observaron corales quebrados, grietas en la estructura arrecifal y desplome del frente del arrecife. La superposición de arrecifes fósiles levantados indica que estos procesos se vienen dando hace mucho tiempo, cabe resaltar que tanto Limón como Cahuita y Puerto Viejo han crecido sobre arrecifes fósiles (Cortés et al., 1994).

A partir de recorridos de campo durante marzo y mayo de 2015, se logró observar en el Parque Nacional Cahuita y el Refugio Nacional Mixto de Vida Silvestre Gandoca-Manzanillo en el Caribe Sur de Costa Rica, como la plataforma calcárea expuesta por los movimientos cosísmicos del Terremoto de Limón está siendo erosionada de manera intensa en la actualidad, probablemente por un proceso de relajación postsísmica del bloque anteriormente levantado (figura 5). 
Figura 5. Erosión costera en el Parque Nacional Cahuita, nótese los árboles muertos y caídos por el efecto del ascenso del nivel del mar. Marzo, 2015.

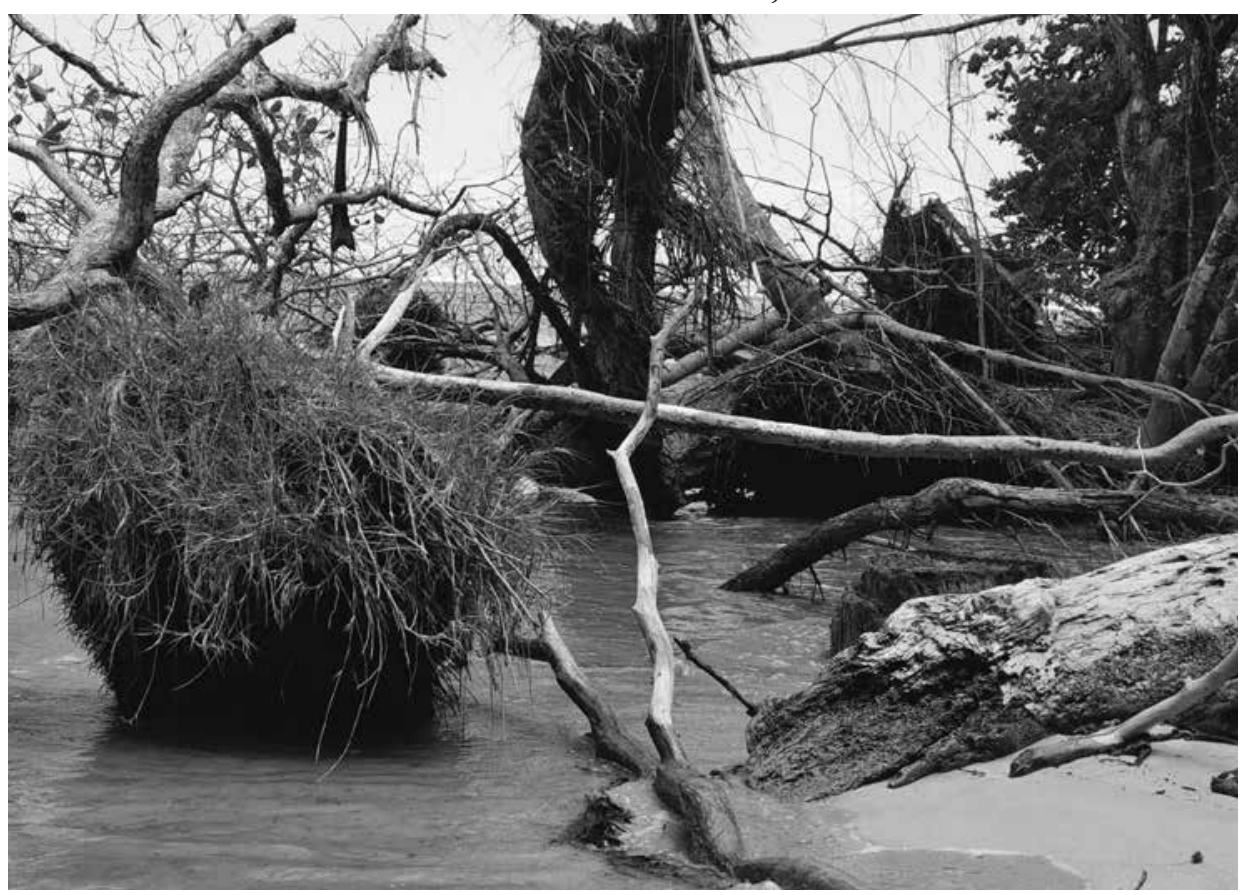

Fuente: Quesada, A, tomada el 8 de marzo del 2015.

La licuefacción fue uno de los procesos generados a raíz del Terremoto de Limón con una importante afectación en términos de extensión espacial, este se concibe como una transformación del material granular de un sólido hacia un líquido debido a un incremento en la presión de los poros. Se asocia con sismos, suelos saturados, suelos arenosos/limosos, colapsos estructurales espectaculares, deslizamientos submarinos y fallos en escombreras (Goudie, 2004).

Unos $3000 \mathrm{~km}^{2}$, aproximadamente, se vieron afectados por la licuefacción de los suelos, dicho proceso se presentó en tierras bajas del sector oriental del litoral Caribe costarricense, con especial actividad en los cordones litorales, barras arenosas playeras, contornos de los estuarios, canales y lagunas litorales; formas del relieve donde la deformación y movimiento se desarrollaron con mayor fuerza gracias a los materiales 
Adolfo Quesada Román. Impactos geomorfológicos del terremoto de Limón (1991; ms=7.5) y

consideraciones para la prevención de riesgos asociados en Costa Rica

DOI: http://dx.doi.org/10.15359/rgac.1-56.4

sin consolidación que forman estas morfologías, lo que generó una intensa fracturación del suelo (Mora y Yasuda, 1994).

Los procesos de licuefacción tuvieron relación con el colapso de cinco puentes, varios de ellos de pequeñas dimensiones con severos daños $\mathrm{y}$ ocho puentes de ferrocarril se asentaron o sufrieron un deterioro parcial; además un total de $80 \mathrm{~km}$ de pavimento y relleno de carretera fueron destruidos impidiendo el tránsito de vehículos (Sauter, 1994). En un futuro evento sísmico semejante al de 1991, es de esperarse una respuesta de licuefacción similar o mayor, debido a que no fue evidente que el diseño de las nuevas estructuras (puentes reconstruidos especialmente) las haga capaces de soportar un suceso similar (Mora y Yasuda, 1994).

Es importante reconocer que las zonas afectadas por licuefacción durante 1991, se relacionan con poblados que son cabeceras cantonales o que tienen una importante densidad poblacional, ejemplo de esto son Bribri, Sixaola, Puerto Viejo y Cahuita en Talamanca, Pandora y Limón en el cantón de Limón y el centro urbano de Matina. Además, estas regiones se relacionan con zonas dedicadas al cultivo del banano, el turismo y centros de servicios bancarios, salud, y educación importantes que de volverse a presentar dicha dinámica en un evento sísmico futuro, representarían pérdidas económicas importantes.

Los procesos de ladera o deslizamientos impactaron, aproximadamente, $2000 \mathrm{~km}^{2}$, siendo las áreas más afectadas las cuencas altas de los ríos Estrella, Banano y Bananito y Chirripó, donde se presentaron deslizamientos espectaculares por su dimensión y extensión (Mora y Mora, 1994). Los términos para nombrar a los procesos que se dan en las laderas y están regidos por la gravedad derivan del inglés, la traducción al español a veces genera confusión, por lo que reconoce varias formas: procesos de ladera (slope processes), procesos gravitacionales (gravitational processes), procesos de remoción en masa (mass movement processes) o deslizamientos (landslides); todos ellos correctos (Alcántara-Ayala, 2000).

Los diferentes procesos de ladera se clasifican según las siguientes clases (UNESCO Working Party on World Landslide Inventory (WP/ WLI 1990); Cruden y Varnes 1996; Alcántara-Ayala 2000; Huggett 2007) en: Deslizamientos, Caídas o Desprendimientos, Volteos, Flujos, Expansión lateral, Movimientos complejos. Los materiales que componen los tipos de procesos de remoción en masa se dividen según el tipo 
de material en: Roca, Detritos (partículas mayores a $2 \mathrm{~mm}$ ) y Suelos (partículas menores a $2 \mathrm{~mm}$ ).

Los sitios más afectados por procesos gravitacionales debido al Terremoto de Limón se componen de laderas formadas de rocas sedimentarias e intrusivas del Terciario con diversos grados de meteorización, estas áreas de máxima incidencia, geomorfológicamente coinciden con áreas que poseen altos valores del Índice de Relieve Relativo (energía del relieve), donde la mayoría de los deslizamientos ocasionaron el desprendimiento y movilización de los horizontes del suelo residual y roca alterada donde su espesor nunca superó los 10 m (Mora y Mora, 1994).

El hecho de relacionar solamente el Índice de Relieve Relativo o Energía del Relieve con las áreas de mayor afectación limita la posible relación con otras variables morfométricas, la incidencia de las estructuras disyuntivas (fracturas, fallas, alineamientos tectónicos), la pluviosidad de la región y el grado de meteorización de los sustratos sean estos de roca, detritos o suelos.

Se infiere que otras variables morfométricas como la densidad de la disección (concentración de ríos en un área específica), la profundidad de la disección (altura relativa entre la ruptura de pendiente más representativa y el talweg), la erosión total (densidad de curvas de nivel en un espacio definido) tienen valores altos en sus métricas, todas ellas potenciadas por la inclinación del terreno superior a $35^{\circ}$ y la presencia de sustratos sedimentarios del Terciario en las áreas más afectadas, relacionadas con las cabeceras de los ríos Estrella, Banano y Bananito y Chirripó.

De acuerdo con Mora y Mora (1994) los deslizamientos se clasificaron como: Deslizamientos superficiales del horizonte regolítico (69\%), Desprendimiento y deslizamiento de masas rocosas (20\%), Deslizamientos traslacionales de masas de suelo y rocas ( $8 \%$ ), y Deslizamientos traslacionales de masas compuestas predominantemente por suelos (3\%).

El estudio de Hernández et al. (1992) determinó un total de 1834 deslizamientos provocados por el terremoto, los cuales variaron de tamaño en función de su localización (litología, pendientes, grado de meteorización y pluviosidad). Las áreas que presentaron cada uno de los procesos de ladera cosísmicos variaron entre $3157 \mathrm{~m}^{2}$ y $19.15 \mathrm{~km}^{2}$, donde se vieron afectadas grandes zonas de bosque en las laderas que descienden desde la Cordillera de Talamanca hacia el mar Caribe. 
Adolfo Quesada Román. Impactos geomorfológicos del terremoto de Limón (1991; ms=7.5) y

consideraciones para la prevención de riesgos asociados en Costa Rica

DOI: http://dx.doi.org/10.15359/rgac.1-56.4

Los deslizamientos aumentaron con las lluvias de alta intensidad de los meses subsiguientes (de mayo a agosto), las tormentas ocurridas después del terremoto fueron superiores al promedio, donde los procesos de ladera asociados al terremoto sumados a la alta precipitación produjeron grandes inundaciones y un arrastre inusual de sedimentos en las cuencas de la zona (Amador et al., 1994).

Esto ocasionó una aceleración en la erosión y la generación de avalanchas de lodo y rocas, del mismo modo, se produjeron represamientos en los cauces fluviales que al romperse incrementaron los caudales y generaron grandes avenidas provocando daños adicionales a la población, líneas vitales y actividades productivas en las regiones bajas (Mora y Mora, 1994).

Los deslizamientos junto a las inundaciones son las amenazas naturales más recurrentes en Costa Rica. Las pérdidas económicas que generan estos procesos, le representan al Estado todos los años gastos en reparación de caminos, puentes y otras vías vitales (como cableado eléctrico, acueductos y poliductos). Además, afectan la propiedad privada, la vida humana y disminuyen el desarrollo social de regiones como la Huetar Norte, Huetar Caribe y Brunca especialmente, ya que el dinero destinado para el mejoramiento de la salud, educación e infraestructura pública y el sector agropecuario deben desviarse para atender desastres cada vez más frecuentes.

\section{Conclusiones}

El terremoto de Limón de 1991, fue una importate enseñanza para Costa Rica en términos de gestión de una emergencia de gran magnitud. Tanto desde el Gobierno Central como desde la CNE se activó todo un sistema de asistencia humanitaria y rehabilitación de infraestructura. También, se implementaron medidas de prevención y mitigación a futuro por medio de la observación de la actividad sísmica, la capacitación de los comités locales, un plan de vigilancia de cuencas, el dragado y construcción de diques, la actualización del Código Sísmico (actualmente se cuenta con una cuarta versión del año 2010) así como el Reglamento de Cimentaciones, y se desarrolló el atlas de amenazas naturales para todo el país.

No obstante, quedan actividades por hacer en términos de prevención y mitigación de la amenaza sísmica en el país, por lo que es importante el monitoreo constante de las reconocidas zonas sismogénicas (siempre 
activa por el proceso de subducción y la cercanía con un punto triple), además se requieren mejores estudios macrosísmicos para determinar las probables intensidades que se pueden presentar ante diferentes escenarios donde intervengan variables geofísicas, tectónicas, litológicas, geomorfológicas y climatológicas; debido a la clara conexión multiamenaza de los sismos con otros peligros, como lo son sus efectos asociados con la activación de deslizamientos, lo cual por ejemplo podría represar ríos que, posteriormente, generarían flujos de lodo o, pueden incidir en el desarrollo de inundaciones debido a la modificación de la carga de los cursos fluviales.

Es importante la implementación de tecnologías de alta precisión como lo son las técnicas geodésicas, el LiDAR (un acrónimo del inglés Light Detection and Ranging), el radar y otras, para generar cartografía con mejor resolución de las áreas susceptibles a presentar deslizamientos durante un terremoto o lluvias extraordinarias (disparadores de procesos de ladera por excelencia). Estos insumos, a su vez, son de vital ayuda para realizar mapas de flujos de lodo e inundaciones, procesos naturales que pueden terminar siendo peligrosos para la población y tienen relación con la amenaza sísmica.

El desarrollo de sistemas de alerta en áreas de reconocida implicación de amenazas naturales es vital en el país, ya existen un número importante de deslizamientos, volcanes y ciertas cuencas hidrográficas con estos instrumentos; sin embargo, el sistema nacional de monitoreo de áreas peligrosas debe ser ampliado, ya que esta herramienta permite la integración de los habitantes de estas regiones en conjunto con los actores institucionales para tomar acciones preventivas y de atención de la emergencia a escala nacional, regional y local. Debe darse especial vigilancia a unidades geográficas de importante densidad poblacional como el Valle Central donde cantones como Desamparados, San José, Alajuela, Cartago, Aserrí, La Unión, Heredia, Alajuelita, Goicoechea y Curridabat tienen un número de registros de desastres en las últimas décadas.

La gestión del riesgo de desastres debe ser un eje transversal en el ordenamiento territorial, para ello debe revisarse el Índice de Fragilidad Ambiental (IFA) de Geoaptitud el cual contempla: 1) el factor de estabilidad de laderas (deslizamientos) y 2) IFA de amenazas naturales, que busca representar la exposición a las inundaciones, licuefacción, vulcanismo, sismos y tsunami, entre otras variables. Se deben realizar estudios a detalle 
Adolfo Quesada Román. Impactos geomorfológicos del terremoto de Limón (1991; ms=7.5) y

consideraciones para la prevención de riesgos asociados en Costa Rica

DOI: http://dx.doi.org/10.15359/rgac.1-56.4

en escalas a de 1:50.000 o superiores, para determinar el verdadero alcance de las amenazas naturales y sus periodos de recurrencia.

De momento, estos índices son estáticos y de aplicación local o regional, por lo que debería modificarse para aplicar mayor dinamismo a estos estudios y permitir el monitoreo a través del tiempo. Los algoritmos para su cálculo deben ser modificados para una mejor compresión de la geodinámica interna y externa a diferentes escalas temporales y espaciales de los municipios del país. Asimismo, cabe resaltar que el hecho de incorporar los IFA en los planes reguladores no asegura que los tomadores de decisiones estén protegiendo los recursos naturales o que se mitiguen los riesgos naturales que afectan los municipios.

\section{Referencias}

Alcántara-Ayala, I. (2000). Landslides: ¿deslizamientos o movimiento del terreno? Definición, clasificaciones y terminología. Boletín de Investigaciones Geográficas, Instituto de Geografía-UNAM, 41; 7-25.

Amador. J.A., Chacón, R.E. y Lizano, O.G. (1994). Estudio de efectos geofísicos del terremoto de Limón mediante percepción remota y análisis hidrometeorológico. Revista Geológica de América Central, Volumen Especial del Terremoto de Limón: (pp. 153-170).

Bergoeing, J.P. (2007). Geomorfología de Costa Rica. Editorial Librería Francesa. San José, Costa Rica. (p. 328).

Bergoeing, J.P. (2013). Paisajes fluviales de Costa Rica. Editorial Tecnológica. San José, Costa Rica. (p. 80).

Berstch, F. (1995). La fertilidad de los suelos y su manejo. Asociación Costarricense de la Ciencia del Suelo. San José, Costa Rica. (p. 157). Bolaños, R., Watson, V. y Tosi, J. (2005). Mapa Ecológico de Costa Rica (Zonas de Vida), según el sistema de clasificación de zonas de vida del mundo de L.R. Holdridge). Escala 1:750 000. Centro Científico Tropical. San José, Costa Rica.

Boschini, I.M. y Montero, W. (1994). Sismicidad histórica e instrumental del Caribe de Costa Rica. Revista Geológica de América Central, Volumen Especial del Terremoto de Limón. (pp. 65-72).

Buol, S., Hole, F. y McCracken, R. (2008). Génesis y Clasificación de los suelos. 2da. Edición. Editorial Trillas. Ciudad de México, México. (p. 417). 
Cortés, J., Soto, R. y Jiménez, C. (1994). Efectos ecológicos del terremoto de Limón. Revista Geológica de América Central, Volumen Especial del Terremoto de Limón. (pp. 187-192).

Cruden, D.M. y Varnes, D.J. (1996). Landslides types and processes. Chapter 3: "Landslides Investigation and Mitigation". Transportation Research Board. National Research Council, Special Report. Número 247.

Denyer, P., Arias, O. y Personius, S. (1994a). Efecto tectónico del terremoto de Limón. Revista Geológica de América Central, Volumen Especial del Terremoto de Limón. (pp. 39-52).

Denyer, P., Personius, S. y Arias, O. (1994b). Generalidades sobre los efectos geológicos del terremoto de Limón. Revista Geológica de América Central, Volumen Especial del Terremoto de Limón. (pp. 39-52).

Denyer, P. y Alvarado G.E. (2007). Mapa geológico de Costa Rica. San José, Costa Rica: Librería Francesa. Escala 1:400 000.

Fernández, J.A., Botazzi, G., Barboza, G. y Astorga, A. (1994). Tectónica y estratigrafía de la Cuenca Limón Sur. Revista Geológica de América Central, Volumen Especial del Terremoto de Limón. (pp. 15-28).

Goes, S. y Schwartz, S.Y. (1991). Rupture process of the April 22, 1991 valle de la Estrella, Costa Rica, eartquake from teleseismic body waves (abstract). Fall AGU Meeting, Supplement to EOS: 301.

Goudie, A. (2004). Encyclopedia of Geomorphology. Routledge, Taylor \& Francis Group. England. (p. 1156).

Güendel, F., Montero, C., Gonzalez, V., Segura, J., Fernández, E., De Obaldía, F., Rojas, D., Rodríguez, H., Mata, A., Van der Laat, R., Barboza, V., Barrantes, O., Marino, T., McNally, K.C. (1991). Mainshock-aftershock sequence associated with the Costa Rica earthquake (Ms 7.5) of April 22, 1991 (abs.) Fall AGU Meeting, Supplement to EOS: 301.

Hernández, G., Vahrson, W. y Ruiz, A. (1992). Deslizamientos producto del terremoto (4-22-91)/Landslides produced by the 22 Apr 1991 earthquake. Mapa publicado. Escuela de Ciencias Geográficas. Universidad Nacional de Costa Rica.

Huggett, R. (2007). Fundamentals of Geomorphology. Routledge. New York, Estados Unidos. (p. 458). 
Adolfo Quesada Román. Impactos geomorfológicos del terremoto de Limón (1991; ms=7.5) y

consideraciones para la prevención de riesgos asociados en Costa Rica

DOI: http://dx.doi.org/10.15359/rgac.1-56.4

ITCR (Instituto Tecnológico de Costa Rica). (2014). Atlas Digital de Costa Rica. Cartago, Costa Rica.

Lugo, J. (2011). Diccionario Geomorfológico. Instituto de Geografía, UNAM. Ciudad de México, México. (p. 479).

Marshall, J. (2007). "The Geomorphology and Physiographic Provinces of Central America”. En: Bundschuc y Alvarado (Eds) (2007). Central América: Geology, Resources and hazards. Taylor \& Francis. (p. 1436).

Montero, W., Ponce, L., Pardo, M., Dominguez, J., Boschini, I., Rojas, W., Suarez, G. y Camacho, E. (1991). The Limon earthquake of April 22, 1991 (Ms = 7.5), seismicity, focal mechanism and tectonic implications (abs). Fall AGU Meeting, Supplement to EOS: 301.

Mora, S. y Mora, R. (1994). Los deslizamientos causados por el Terremoto de Limón: factores de control y comparación con otros eventos en Costa Rica. Revista Geológica de América Central, Volumen Especial del Terremoto de Limón. (pp. 139-152).

Mora, S. y Yasuda, S. (1994). Licuefacción de suelos y fenómenos asociados durante el Terremoto de Limón. Revista Geológica de América Central, Volumen Especial del Terremoto de Limón. (pp. 121-132).

Mora, S. y Valverde, R. (2005). La Geología: procesos de la dinámica interna y externa. 2da edición. Editorial Tecnológica de Costa Rica. Cartago, Costa Rica. (p. 376).

Morales, L.D. (1994). Daños causados por el Terremoto de Limón: pérdidas y medidas de mitigación. Revista Geológica de América Central, Volumen Especial del Terremoto de Limón. (pp. 201-210).

Obaldía, F., Marino, T., Van Der Laat, R., Malavassi, E., y Hernández, F. (1991). Levantamiento cosísmico asociado al terremoto del 22 de abril de 1991, Ms=7.5 Valle de La Estrella, Limón, Costa Rica. Observatorio Vulcanológico y Sismológico de Costa Rica, Universidad Nacional. Heredia, Costa Rica.

Quesada, R. (2007). Los Bosques de Costa Rica. IX Congreso Nacional de Ciencias. Instituto Tecnológico de Costa Rica. Cartago, Costa Rica. (p. 16).

Rojas, W. (1991). El terremoto del 22 de abril de 1991 en la región Caribe y la sismicidad asociada en el de abril, 1991. Laboratorio de Sismología, Universidad de Costa Rica. San José, Costa Rica. (p. 65). 
Sauter, F. (1994). Evaluación de daños en puentes y otras estructuras civiles causados por el terremoto de Limón. Revista Geológica de América Central, Volumen Especial del Terremoto de Limón. (pp. 171-186).

Solano, J. y Villalobos, R. (2001). Aspectos Fisiográficos aplicados a un bosquejo de Regionalización Geográfico - Climático de Costa Rica. Tópicos de Meteorología y Oceanografía, 8, 26-39. Instituto Meteorológico Nacional. San José, Costa Rica.

USGS (United States Geological Survey). (2015). Significant Earthquakes of the World: 1991. Recuperado de http://earthquake.usgs.gov/earthquakes/eqarchives/significant/sig_1991.php (último acceso: 3 de setiembre de 2015).

WP/WLI (International Geotechnical Societies UNESCO Working Party on World Landslide Inventory). (1990). A suggested method for reporting a landslide. Bulletin of the International Association of Engineering Geology, 41, 5-12. 